\title{
EPICS UPGRADE OF IPNS ACCELERATOR CONTROL AND DATA ACQUISITION SYSTEM
}

\author{
Garrett Rinehart, Gerald McMichael, ANL, Argonne, IL 60439, USA
}

\begin{abstract}
The Intense Pulsed Neutron Source (IPNS) facility operates a $450 \mathrm{MeV}$ proton accelerator to generate neutrons for science. The accelerator data acquisition and control system was growing increasingly stagnant and difficult to maintain due to its reliance on computer technology from the 1970's. A concerted effort was put forth to upgrade that system to a new, distributed system utilizing the Experimental Physics and Industrial Control System (EPICS) [1]. There were several issues that needed to be addressed in order to accomplish this transition. The computer system has to be synchronized with the $30 \mathrm{~Hz}$ repetition rate of the accelerator. The transition from Computer Aided Measurement And Control (CAMAC) to Versa Module Europa (VME) requires that different connectors be installed for all of the I/O cabling and, in some cases, special hardware modifications. Completely new operator displays and controls had to be developed. The IPNS facility operates year-round with only a week or two between runs and roughly one month off in the summer and one in the winter. This paper describes the transition to the present system and how the upgrades were accomplished in an incremental fashion during the short shutdown periods.
\end{abstract}

This work is supported by the U.S. Department of Energy under contract number W-31-109-ENG-38

\section{INTRODUCTION}

The IPNS accelerator system [2] includes three accelerators. The first stage is an $\mathrm{H}$ ion source in a high voltage enclosure connected to the terminal of a $750 \mathrm{keV}$ Cockcroft-Walton generator. The $750 \mathrm{keV} \mathrm{H}$ is injected into a $50 \mathrm{MeV}$ Alvarez linac, which in turn injects beam (after stripping to protons in a thin carbon foil) into a 450 $\mathrm{MeV}$ rapid cycling synchrotron. The preaccelerator and linac began operation in the early 1960's as the injector for the $12.5 \mathrm{GeV}$ Zero Gradient Synchrotron and have now operated another twenty years as part of the IPNS accelerator system. The output beam from the synchrotron is directed to a depleted-uranium target to produce intense pulses of neutrons which are then moderated and directed through 12 separate neutron beamlines to neutronscattering instruments. IPNS operates at a pulse repetition rate of 30 pulses per second $(30 \mathrm{~Hz})$ with the output proton beam pulse synchronized to within a couple of microseconds to the rotating neutron choppers.

In 1992, IPNS was operating with a large portion of the accelerator control and data acquisition system being handled by two Data General computers dating back to before facility start-up in 1981. Most interfacing to the accelerator was done via Computer Aided Measurement And Control (CAMAC) crates and modules, with the remainder of it through facility-built devices. Much of the operator interface was via digital readouts (multi-digit LED displays) in facility-built modules interfaced directly to an extended Data General backplane. The console "terminal" was a specialized keyboard and monitor, also connected directly to the Data General. An upgrade was both necessary and desirable. The Data General computers were "mainframe" type units which were comprised of stacks of 15" square printed circuit boards, containing TTL logic. The connections to the backplane had become so unreliable that we had discontinued the practice of shutting the systems down between accelerator run cycles because the thermal cycling alone would cause the boards to unseat from the backplane. Spares were no longer available for parts of both the computers and the CAMAC systems and a much better operator interface was needed.

The Experimental Physics and Industrial Control System (EPICS) was being heavily developed by and for the Advanceed Photon Source (APS) at that time. The decision was made to migrate IPNS to EPICS.

\section{CHALLENGES}

\subsection{Short Transition Times}

The IPNS facility generally operates with only a week or two between runs. It is also shut down for one month in the summer and one month in the winter. These short down-times make implementing changes in the control system difficult. Changes to hardware, including cabling, have to be made quickly and tested thoroughly with enough time left over to remedy any problems, even if that means reversing the change.

\subsection{CAMAC}

Much of the control system utilized CAMAC crates and modules. CAMAC was used to control magnets and beamline device positioning. It was used to write to a remote digital status panel, as well as read back analog values for magnet currents, beamline toroids, loss monitors, and target cooling system paramters. In addition, it read binary status of beam gates, and many other digital indicators. Unfortunately, CAMAC was not originally supported by EPICS.

\subsection{Trip Function}

Each monitored channel in the old IPNS control system was checked against up to four limits; two high and two low. In each direction, the first limit was an alarm to 
notify the operator of a problem. The second limit was a trip which would automatically shut down the machine.

EPICS incorporates two high and two low alarms like the old system and allows for different severity levels with each one. Unfortunately, it did not allow for taking any action by itself. Another program (ie: "ALH", the alarm handler) could be set up to monitor the state of a given record and take action under certain circumstances. However, there would be no way to guarantee that the program would always be running or that the network connection between the monitoring computer and the IOC would be operational.

\section{$2.430 \mathrm{~Hz}$ Repetition Rate}

The IPNS accelerator operates with a repetition rate of $30 \mathrm{~Hz}$. Some of the accelerator systems have to be monitored each machine cycle. The EPICS databases, which typically run asynchronously at no more than $10 \mathrm{~Hz}$, had to be made to run more often, and to run synchronously with an external event.

\subsection{Operator Transition}

In order to maintain the long-standing record for reliability that the IPNS has, it is imperative that the operators understand and are comfortable with the controls and readouts they are given. There is a world of difference between the old and the new and the transition had to be made carefully, lest reliability suffer from operator confusion.

\section{IMPLEMENTATION}

\subsection{Short Transition Times}

Upgrades were made in an incremental fashion. Each system was broken down into the smallest parts that could be separated. Then each small, separate part was upgraded, one at a time. This made each individual hardware and software change as easy as possible. It also made it as easy as possible to test the modifications and affect repair where necessary, in the time available.

\subsection{CAMAC}

TJNAF (CEBAF at the time) had a legacy CAMAC system similar to ours, and they wrote a driver for a VME/CAMAC interface board. [3] With it, all of our original CAMAC I/O remained intact, initially, and only the host computer was changed. EPICS databases were set up to handle all of the $\mathrm{I} / \mathrm{O}$ and the minor cabling change from the old computer to the new VME Input/Output Controller (IOC) was made between run cycles. The IOC does the work the Data General used to do and a new Unix workstation was installed to replace the old specialpurpose terminal for the operator interface. The switch was timed to be made just before the system went through its rigorous quarterly test proceedure. After some problems pointed out by the test were fixed, the system was ready.

\subsection{Trip Function}

Modifications to standard records were made to create trip records. These function just as their standard counterparts do, with the addition of trip capability. Any alarm severity above "Minor" causes a trip link to be processed in the EPICS database. All of the trip records link to one record that actually causes the machine to be shut down by writing a bit to the shutdown chassis. A reset by the operator simply unsets that bit

\section{$3.430 \mathrm{~Hz}$ Repetition Rate}

A digital I/O board is added to each IOC requiring synchronization. The beam pulse triggers the digital I/O board, which interrupts the CPU. The CPU then executes an EPICS event record. The event record posts a software event, which triggers any record in the IOC that is set for that event. A simple counting scheme and other event records are used so that certain records can be set to run synchronously with every beam pulse, or every nth beam pulse. For example, a counter that increments every beam pulse and rolls over every third time will cause its associated event to post every three beam pulses.

\subsection{Operator Transition}

A logical approach was taken to creating computer displays. Items of a similar nature or pertaining to the same subsystem were put on the same displays. Each display is accessed from a small master display which contains a group of menu buttons. Since everything is accessed in the same manner and from the one master display, the operators quickly became accustomed to it.

The other main operator interface type was that of digital LED readouts working in conjunction with sets of buttons and knobs to select and control a given channel. The digital readouts were replaced with LCD touchscreens, and virtual buttons on the touch-screens replaced the real buttons. The operators prefered the control they had with the knobs, so the knobs were kept.

\section{PRESENT SYSTEM}

A block diagram of the present system is given in Figure 1.

\subsection{Improved Operator Interfaces}

The new computer displays can be easily modified to accomodate new parameters or combinations of parameters.

The touch-screens, in place of actual buttons and LED readouts, make it possible to reorder buttons, add buttons, and customize the display format.

The beam profile and position at the target used to be represented as a rectangle on the computer screen. Now they are represented it as two curves, each comprising 32 intensity values.

Striptool, a virtual strip-chart application for EPICS, allows us to continuously monitor trends in real-time. When used to monitor channels that are sensative to beam tuning, such as ring vacuum, this feature provides the operators with immediate qualitative feedback as they 
tune the machine. At a glance, they can tell whether the machine is running better or worse, without having to remember previous numbers as they would with an ordinary readout.

\subsection{Higher Channel Count}

Before the upgrade, the computer system monitored approximately 275 analog channels and roughly 150 binary channels. It also controlled devices through about 20 analog outputs and 8 digital outputs. In addition to those, it also wrote over 100 bits of status to an annunciator panel in a remote location.

The upgraded system handles all of the original channels, plus a great deal more. It now reads about 15 more analog channels, and 110 more binary channels. It also has an additional 40 analog control outputs and a few additional binary control outputs. In addition to that, the new system also makes use of RS-232 serial ports to communicate with several new devices. In order to accomodate the additional channels, where we used to have only one computer display, we now use seven, with an additional four replacing LED readouts.

\subsection{Improved Data Logging}

Utilizing the Self Describing Data Set (SDDS) toolkit, the logging, reporting, and analyzing oportunities available with EPICS far exceed those available with the limited utilities of the old computer system.

The reporting and plotting that used to have to be done manually on a daily basis is now handled automatically. In some cases, it has even become paperless via e-mail.

\section{CONCLUSIONS}

Upgrading our control system in an incremental fashion has worked very well for us. It has, in fact, become an ongoing process. Further upgrades are made on an almost continuous basis. Near-term plans call for the addition of over 120 new analog channels for the linac. To avoid becoming stagnant in computer technology as we did with the Data General computers, we have begun a routine upgrade of workstations. They will be replaced at a rate of about one per year. The old, facility-built function generators which drive pulsed tuning magnets in the synchrotron are to be replaced with Commercial Off the Shelf (COTS) VME modules.

\section{REFERENCES}

[1] http://www.aps.anl.gov/epics

[2] http://hemlock.pns.anl.gov/operations

[3] W. Watson, "The CEBAF Control System", IEEE PAC 95, 95CH35843, 2167, 1995

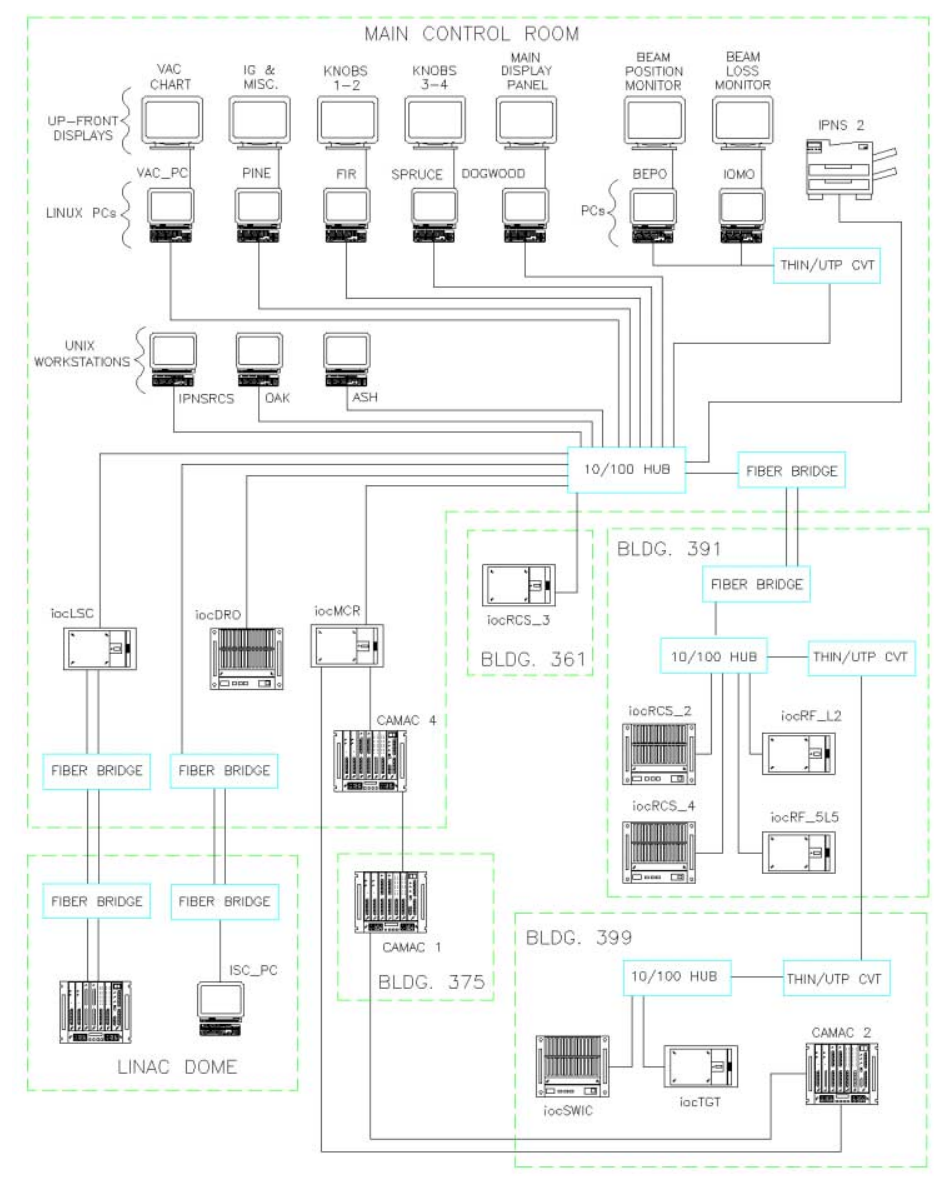

Figure 1 